\title{
The Evolution of Informed Consent: Failures of the Current System of Human Protection
}

\author{
David A. Lenrow, MD* and Larry H. Chou, MD\#
}

The reliance of subjects and researchers on the current regulatory scheme for human subject protection is misplaced. Investigators often assume that compliance with the requirements of the Common Rule, including Institutional Review Board approval of their research project, adequately fulfills the spirit of the federal regulation and protects them from liability for lack of adequate informed consent. Subjects and physicians referring subjects, believe that institutional approval of studies assures that they are scientifically valid and ethical. Unfortunately the current scheme of human research protection has failed to fulfill the spirit of the Nuremberg Code and The Belmont Report. Not only are large numbers of studies left out of the current protection scheme but the scheme is also flawed on several levels. This leaves researchers at risk for liability and subjects may have their right to autonomy violated even while the requirements of the current regulatory scheme are satisfied. It is time for a wholesale restructuring of human subject protection. If physician researchers ignore the deficits of the current system we will lose control and input into the formulation of the new scheme.

Keywords: Institutional Review Boards, Informed Consent, Human Subject Protection
Research institutions, including academic institutions, rely on the Federal Regulatory Scheme embodied in the Common Rule to protect human research subjects. The spirit of this scheme was to implement the tenets of the Nuremberg Code (1) and The Belmont Report (2). Research must be ethical and voluntary; fully informed consent is required for all human research (1-3). Unfortunately the regulatory scheme is vague and implementation is in the hands of local Institutional Review Boards (IRBs) who are given little guidance by the regulations. The composition of the IRBs is in itself in conflict with the spirit of the goals of human subject protection and the requirements of informed consent in research. There is no federal oversight of review boards or of the method of obtaining informed consent. The current scheme leaves out an increasing number of human subjects. As funding of research has shifted to the private sector larger numbers of subjects fall outside even the limited protections of the Common Rule. This leaves investigators and subjects with inadequate protection. Compliance with the current regulations does not

From the Department of Rehabilitation Medicine, University of Pennsylvania, Philadelphia, Pennsylvania. *Dr. Lenrow is Vice Chairman of Clinical Services in the Department of Rehabilitation Medicine. "Dr. Chou is the director of physical medicine and rehabilitation services. Address Correspondence: David Lenrow, MD, 5th floor Gates Bldg, 3400 Spruce Street, Philadelphia, PA 19104. E-mail: dlenrow@mail.med.upenn.edu ensure subject protection or investigator compliance with the requirements of informed consent.

\section{CURRENT SYSTEM}

Investigators currently submit their research protocols to IRBs for approval. The role of the IRB is two-fold. One is to evaluate the scientific value of the project and balance that with the risk to the subjects. That is an appropriate task for individuals with knowledge of the science being investigated. The project must be understood and the benefit to society and science, as well as, the risk to the subjects must be compared. The current IRBs should have the ability to fulfill that role. They are composed primarily of members from the parent institution, which in most cases is an academic institution with investigators on the IRB board. Even if we ignore the conflict of interest apparent in having insiders approve studies leading to funding to benefit the parent institution (often the employer of the board members and researchers) the second role of he IRB is not met. The second role of the IRB is to ensure that the rights and autonomy of the subjects are protected. They are to evaluate the adequacy of informed consent.

\section{FLAWS IN REGULATION}

A brief detour to the required disclosure of informed consent in research versus treatment makes clear why IRBs are inadequate to insure compliance with informed con- 
sent. This was discussed in greater detail in the first part of this series. The standard for disclosure in research is stricter than that in treatment. Beneficence of physicians in treatment and mixed motives and unknown outcomes in research, justify these differences. The standard of disclosure is that of the reasonable subject. That means disclosure of all information that the reasonable subject would require to make an informed decision regarding participation in the study. The IRBs are not the community of potential research subjects; they are not synonymous with the reasonable subject. The federal regulations only require one outside member on an IRB with a minimum of five members (4). The current scheme of research regulation does not even meet the standard of disclosure required in treatment and certainly does not meet the standard required in research. We have professionals on the IRB determining the adequacy of disclosure in informed consent. That is the professional standard of disclosure, which has been found to be inadequate by many jurisdictions in treatment. The enumerated standards in research are clearly stricter and therefore not met. Only individuals similarly situated to potential subjects could determine if the extent of disclosure was adequate to facilitate fully informed consent. We need to rethink the make up of institutional review boards to comply with the Nuremberg Code and The Belmont Report.

The reasonable subject standard of disclosure has been applied by the courts and suggested by ethicists. Why do we not follow this rule with IRBs? It is interesting to note that in other countries IRBs or their equivalent are made up of a much larger percentage of community members. In the Dutch system 50\% of the IRB is made up of nonscientist community members (5). Australia has a similar system, requiring a broader representation of laypersons on their review boards than in the U.S. (6). The Department of Health and Human Services, Office of Inspector General has suggested having a large percentage of the review board consist of laypersons from the community (7). The board is then better able to assess what the reasonable subject would require as disclosure to make an informed decision, regarding whether to agree to participate in the study or not. The criticism of these community IRBs is that they cannot understand the science or the value of the research. In a nutshell this exemplifies the problem with the current system and the prevalent attitude in the scientific community. If the potential subject cannot at some level understand the science, as well as the risks and benefits of the study, we can never obtain informed consent. The subjects understanding should not be viewed as an obstacle to scientific and medical progress. Subjects' understanding and informed consent must be an integral part of human subject research. It is required to maintain individual autonomy and the integrity of the medical and scientific community.

If the criticism is that the lay public may accept scientific studies that have unacceptable scientific validity, then one could argue that review boards, which are composed of predominantly community members, may be inadequate. This is a very different argument from asserting that the board could not understand the science enough to evaluate the adequacy of disclosure. Setting up a dual review system would solve the valid concern that unacceptable science may pass by the community review boards. The first review could be by a scientific review board, not unlike our current IRBs. They would evaluate the merit of the study on scientific grounds. If the study passed this first review, only then would it go on to review by the community review board consisting of primarily laypersons. To be valid, the laypersons should be the same make-up as the potential subjects of the experiment. That would allow review from the perspective of the targeted population of subjects. They would determine the adequacy of disclosure and the process of consent. The increased funding available from governmental and private sources has made it more difficult for IRBs to adequately evaluate studies. They are over burdened. The current membership of IRBs is inadequate to represent the interests of subjects $(8,9)$. Changes in the membership of the boards and the addition of supervision, of the process of informed consent, should be instituted.

In 1995 President Clinton created the National Bioethics Advisory Commission. They released a draft report in December of 2000 (11). The report detailed inadequacies in the Federal oversight of human subject research and recommended major changes. The current administration of President George W. Bush and Congress has not yet acted on this report. The recommended changes included (among others):

- Congress should create an independent office to oversee human research. Currently the Office for Human Research Protection (OHRP) is under the Department of Health and Human Services.

- At minimum half of the members of IRBs must be from outside the institution. At minimum half are to be non-scientists.

- All research should be covered, not only federally funded research.

- University review boards should supervise the in- 
formed consent process under certain situations (11).

In the National Bioethics Advisory Commission's (NBAC) draft policy they have extensively outlined the history and reasoning for revamping the current federal scheme of research regulation. The piecemeal current system is failing in many respects. They base much of their newly proposed regulation on the principles set forth in The Belmont Report (12).

The training and role of individuals on IRBs may be inadequate. They are volunteers in most institutions, except for some core staff. Their allegiance lies with their colleagues whose studies they are reviewing. A prevalent attitude is that IRBs are for the protection of the institution, when in reality their role is the protection of the subjects. The ability of an IRB to sufficiently protect the subject's autonomy does not exist in the current system. In a recent study of IRBs, some only spend one or 2 minutes reviewing each study (13). Standardized procedures and uniform oversight and regulation are necessary to protect subjects and investigators. A uniform system will also lead to greater certainty upon which researchers can rely. Standards will be ascertainable and not dependent on the particular IRB or trend in that institution. Presumably this would lead to a learning curve among researchers and facilitate ethical research that comports with the rules of the Nuremberg code and The Belmont report. The result would be less resubmission to IRBs with less work for the boards and quicker approval of research. Clear requirements for informed consent would benefit IRBs, investigators and subjects.

\section{CHANGES IN RESEARCH FUNDING}

The importance of rigid regulation, oversight and protection of human research subjects has become increasingly more urgent. Current experiments in violation of Human Rights spurred most of this legislation, but violations continue to be uncovered even in the most prestigious universities. Increased funding for research has led to increased numbers of research subjects. In 1986 Federal funding for medical and health related research was $\$ 6.9$ billion (14). This figure nearly doubled by 1995 to $\$ 13.4$ billion (14). Only approximately half of that funding went to university research programs (15). An even more threatening change is the increasing privatization of research funding. Private industry funding for research is growing at an even faster rate. During the same period 1986 to 1995 , funding tripled from $\$ 6.2$ to $\$ 18.6$ billion dollars (16). Pharmaceutical company research has exploded in the past 20 years. In
1980 they funded $\$ 1.5$ billion in research. By the year 2000 there was $\$ 22.4$ billion in funding for human research by the pharmaceutical industry (17). Their role on the research front has become a formidable force. The current regulation of research funded by industry only applies to drugs and investigational devices that are not yet approved. Under the current system once approved, they are no longer under the auspices of the FDA or NIH regulatory schemes. Further research projects would not be regulated, i.e. subject to the Common Rule or IRB review.

The dramatic increase in the amount of money available for research has greatly increased the number of investigators in the U.S. The total number of investigators regulated by the FDA rose from 5,500 in 1990 to 25,000 in 1996 (18). There are estimated to be a total of 50,000 investigators in the U.S. as of 2000 (19). This makes the problem of regulation of both federally funded and industry sponsored research an imposing problem. The assumption that the integrity of individual investigators or their sponsoring institutions will insure compliance with research ethics has been proven false by history. Systematic regulation and constraints are necessary, but individual potential subjects must remain empowered to make a free and informed choice regarding participation.

The face of health care has changed dramatically in the last decade. Managed Care Organizations and competition for patients has limited clinical funding available for research (20). The response has been for academic institutions to join forces with industry in research endeavors (21). This has led to a set of conflicts of interest, for most academic institutions, that had not previously been addressed by their review boards or on an institutional basis.

Research sponsored by industry is not limited to academic institutions. The trend is for industry-funded research to be performed outside of academic institutions. Henderson noted that in 1998 only $40 \%$ of industry-sponsored research funding for clinical trials went to academic medical centers (22). In $199180 \%$ of industry funding for clinical trials went to academic medical centers (22). The remainder of the industry-funded research is managed by private organizations.

This places a significant subset of research beyond the regulation of the FDA and the Common Rule regulation of federally funded research. These inadequacies continue to grow as funding of research changes. This changing face of research also brings with it new conflicts of interest between the investigator and the potential subjects. When 
investigators have a financial interest in the outcome of research that should be disclosed to subjects. The permissible degree of personal, financial or other benefit to the investigator is not defined. What was clarified, by the court in Moore is that the potential subjects have a right to be informed of any benefit (23). This includes any potential financial benefit. In practice that is nearly impossible to fulfill. Outside funding sources are disclosed to IRBs and subjects but the implications are often not apparent to the subject. Without understanding informed consent is inadequate even if there was adequate disclosure.

\section{PROPOSED CHANGES IN REGULATION}

The proposed changes by NBAC address some of the inadequacies of the current regulatory scheme. If they are to work they must be clearly defined with central oversight. The IRBs must be either dual boards with layperson review of informed consent or they must be made up of a majority of non-professional members. These changes correct the deficiencies in assuring that informed consent is fully informed, that disclosure is adequate. We must monitor protocols in practice for compliance with appropriate informed consent. Mere disclosure is not enough. Subjects must be able to manipulate the information and give informed consent. Understanding is a requirement.

The changes recommended do not address the voluntariness requirement of informed consent of the Nuremberg Code and embodied in The Belmont Report. Subjects under undue influence or coerced cannot give voluntary consent. The Common Rule recognizes subgroups who require additional protection but does not define or give guidelines to assure consent is voluntary. That is left in the hands of the investigator. IRBs are not involved in the actual process of consent and do not in anyway moderate the interaction between investigator and subject that constitutes informed consent. Informed consent must be an ongoing process. It is not limited to a specific point in time but requires an ongoing discourse between subject and investigator. In treatment trust and beneficence of the physician facilitate consent but in research the motivation of the investigator is not only to cure or help the individual. Often the motivation is to gain knowledge or improve future treatment with little benefit to the individual subject. Relying on the potential treatment of a research protocol is termed the therapeutic misconception (24-26). This trust is misplaced. If subjects are not informed or do not understand the difference between research and treatment investigators put themselves at risk for lawsuits if something goes wrong. The protection of research subjects will also protect the investigator.

\section{CONCLUSION}

We must learn from history that physicians and universities do not adequately police human research. The Doctors Trial (27), Tuskegee (28), and more recently the research deaths at Johns Hopkins and the University of Pennsylvania are examples $(29,30)$. In the latter two instances investigator's ethics and oversight by the universities have been called into question. Lawsuits for failure to obtain adequate informed consent in research are not a thing of he past.

As clinical dollars decrease due to declining reimbursement physicians are pushed to obtain funding for research from industry. This trend is rapidly expanding. This creates new conflicts of interest between investigators and subjects. Many universities have guidelines to deal with these conflicts but we are relying on self-regulation in IRBs to protect the investigator from tort suits and the subject's autonomy from unethical or unknowing researchers. Comprehensive federal regulation of human subject research is necessary. The recent breaches at the University of Pennsylvania and at Johns Hopkins are a result of the failure of the current system. Approval of research is not enough. There must be oversight by a central body with the autonomy necessary to perform independently of funding organizations. There must be ongoing review of human research including ethics education early on in training of investigators.

The current responses to the inadequacies of the system are again only responses to transgressions. We need wholesale restructuring of how human research is regulated at the national level. Web based training of researchers in ethics will not prevent breaches of informed consent. It is time to learn from history and realize that human research requires clear ethical standards. If we continue to delude ourselves we will lose credibility with the public and restructuring of the mechanism of oversight will proceed without physician/investigator input.

The scheme of human subject protection must be all-inclusive and not limited to research that is federally funded or investigational drugs or devices. It must fulfill the goals of the Nuremberg Code and The Belmont Report. The requirements of disclosure; the reasonable subject standard must be met. Methods to assure voluntariness and eliminate or disclose conflicts of interest should be part of the scheme. Individual autonomy does not bend to aca- 
demic freedom or the advancement of science. The proof is in the atrocities committed in the name of science that have led to the current regulatory scheme. The argument that investigators commonly respond with is that regulation of human research is too cumbersome; it slows down the advancement of science. Subverting subjects rights to autonomy is not the answer. The answer is building a system of regulation anew. The current system is under-inclusive, slow and inefficient; it is inadequate. It does not protect investigators or subjects. It is time to stop bemoaning regulation and embrace it as part of human research. If physician investigators lobby for a new efficient and adequate scheme of protection the public, researchers and subjects will all benefit.

\section{REFERENCES}

1. International Military Tribunal. Trials of war criminals before the Nuremberg Military Tribunals under Control Council law no. 10. Washington, D.C.: Government Printing Office, 1950.

2. National Commission For The Protection Of Human Subjects Of Biomedical And Behavioral Research, The Belmont Report: Ethical Principles And Guidelines For The Protection Of Human Subjects Of RESEARCH 3 (1979). Hereinafter The Belmont Report, 44 Fed. Reg. 23192 (1979).

3. $\quad 45$ C.F.R. 46 (2001).

$4 . \quad 45$ C.F.R. $\$ 46.107$ (2001).

5. Menache, A. Healthy Human Volunteers and Informed Consent. Med. Law 2000; 19: 523, 525, citing The Danish Central Scientific Ethical Committee; Collection of Annexes, Copenhagen (1994).

6. Beran, R. The Ethics of Clinical Research and the Conduct of Clinical Drug Trials: International comparisons and Codes of Conduct. Med. Law 2000; 19: $502,516$.

7. Institutional Review Boards: A Time for Reform, OEI01-97-00193 (1998).

8. Woodward B. Challenges to Human Subject Protections in US Medical Research. JAMA 1999; 282: 1947, 1951, citing G. J. Annas, Good as Gold, in Judging Medicine. 1988; 325.

9. McNeill PM. International Trends in Research Regulation: Science as Negotiation in Weisstub DN. (editor) Research on Human Subjects: Ethics, Law and Social Policy. 1988; 245

10. Policy Issues in Research Involving Human Participants, National Bioethics Advisory Commission, December 19, 2000, Draft Report for Public Comment.

11. Brainard J. Panel Proposes New Guidelines for Research With Human Subjects. The Chronicle of Higher Education, January 12, 2001:A24.
12.

13.

44 FR 23192 (1979).

Grob G. Scientific Research: Continued Vigilance Critical to Protecting Human Subjects, Washington DC: General Accounting Office; GAO/HEHS-96-72 (1996).

14. NIH Extramural Data and Trends.http://grants.nih.gov/ grants/award/trends95/ANNOTATE.HTM>.

15. Policy Issues in Research Involving Human Participants, National Bioethics Advisory Commission, December 19, 2000, Draft Report for Public Comment Ch. 1 p. 4.

16. Estimates of National Support for health R\&D by Source or Performer, 1986-1995. Http://grants .nih.gov/grants/award/trends95pdfdocs/fedtabl1.pdf

17. PhRMA (Pharmaceutical Research and Manufacturers of America). 2000. Pharmaceutical Industry Profile 2000. Washington, DC: PhRMA, Http://www. PhRMA.org

18. Valigra L. Rooting Out Rogue Investigators. CenterWatch 1997; 4(7): 1, 5.

19. Policy Issues in Research Involving Human Participants, National Bioethics Advisory Commission, December 19, 2000, Draft Report for Public Comment Ch. 1 p. 4, citing Turbulence and Transition: The Top Stories of 1999. (editorial) CenterWatch 2000; 7(1): $1,5-10$.

20. Dobson RE. The Impact of Managed Care on Clinical Research: A Preliminary Investigation. Health Affairs 1986; 15: 72-89.

21. Gallin JI. Managing the Interface Between Medical Schools Hospitals, and Clinical Research. JAMA 1997; 277: 651-654.

22. Henderson L. Centralized AMCs Growing Steadily. CenterWatch 1999; 6(2): 4-5.

23. Moore v. Regents of the University of California, 793 P.2d 479 (Cal. 1990).

24. Letter from Niels Lynoe, Umea University Sweden to The New England Journal of Medicine, N Engl J Med JM 2001; 344: 460.

25. Appelbaum PS. False Hopes and Best Data: Consent to Research and the Therapeutic Misconception. Hasting Center Report 1987; 17(2): 20-24.

26. Schultz AL. Are Research Subjects Really Informed? 23 West Journal of Medicine 1975; 23: 76-80.

27. United States v. Karl Brandt et al. (case 1). Washington, D.C.: National Archives, November 21, 1946August 20, 1947.

28. Jones JH. Bad Blood: The Tuskegee Syphilis Experiment. New York: Free Press, 1981.

29. Steinbrook R, Protecting Research Subjects-The Crisis at Johns Hopkins. N Engl J Med 2002; 346 (9): 716-720.

30. Kahn JP, Mastroianni AC, Doing Research Well by Doing Right. The Chronicle of Higher Education Feb 15, 2002: B24. 